\title{
US NEUTRINO FACTORY STUDIES
}

S. Ozaki and R.B. PaImer

Brookhaven National Laboratory

M.S. Zisman

Lawrence Berkeley, National Laboratory

\section{CENTER FOR ACCELERATOR PHYSICS}

\author{
BROOKHAVEN NATIONAL LABORATORY \\ BROOKHAVEN SCIENCE ASSOCIATES \\ Under Contract No. DE-AC02-98CH10886 with the \\ UNITED STATES DEPARTMENT OF ENERGY
}

Proc. of "3ri International Workshop on Neutrino Factories Based on Muon Storage Rings", rsukuba, Japan, May 24-30, 2001, (Invited Paper); Nucl. Instru. \& Methods in Phys. Res., A 503 (2003) 20-25. 


\section{$\because \quad$ DISCLAIMER . .}

This report was prepared as an account of work sponsored by an agency of the United States Government. Neither the United States Government nor any agency thereof, nor any of their employees, nor any of their contractors, subcontractors, or their employees, makes any warranty, express or implied, or assumes any legal liability or responsibility for the accuracy, completeness, or usefulness of any information, apparatus, product, or process disclosed, or represents that its use would not infringe privately owned rights. Reference herein to any specific commercial product, process, or service by trade name, trademaxk, manufacturer, or otherwise, does not necessarily constitute or imply its endorsement, recommendation, or favoring by the United States Government or any agency, contractor or subcontractor thereof. The views and opinions of authors expressed herein do not necessarily state or reflect those of the United States Government or any agency, contractor or subcontractor thereof. 


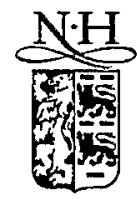

ELSEVIER
Available online at www.sciencedirect.com

science Dratoto

Nuclear Instruments and Methods in Physics Research A 503 (2003) 20-25

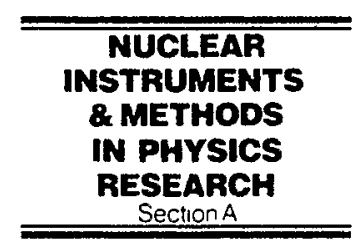

www.elsevier.com/locate/nima

\title{
US neutrino factory studies
}

\author{
S. Ozaki ${ }^{\mathrm{a} *}$, R.B. Palmer ${ }^{\mathrm{a}}$, M.S. Zisman ${ }^{\mathrm{b}}$ \\ ${ }^{a} B N L$, Upton, NY 11973-5000, USA \\ ${ }^{\circ}$ LBNL, Berkeley, CA 94720, USA
}

For the Neutrino Factory and Muon Collider Collaboration

\begin{abstract}
The design and simulated performance of a second feasibility study are presented. The efficiency of producing muons is $\approx 0.17 \mu / \mathrm{p}$ with $24 \mathrm{GeV}$ protons. This study was sponsored by the BNL Director, with BNL site specific driver and layout. It was a follow on to the First Study (http://www.fnal.gov/projects/muon_collider/nu-factory/fermistudy_after_aprillst/) sponsored by the Fermilab Director, with Fermilab site specific driver and layout, and was the main US collaboration conceptual effort during the past year. Other studies, and technical work by the collaboration is reported in other papers.

(C) 2003 Elsevier Science B.V. All rights reserved.
\end{abstract}

Keywords: Neutrino; Muon

\section{Introduction}

A second feasibility study was commissioned by the Brookhaven National Lab Director, John Marburger and was presented to him on May 4, 2001. It was a follow on to the study commissioned by the Fermi Lab Director FNAL specific (Study 1) that was presented in April 2000 [1]. In each study there are site specific parts, the driver and storage ring angle, in particular, that are for the Fermilab location and BNL sites respectively. Otherwise the studies are generic with the primary difference that the second study is aimed at a lower muon energy (to save cost), but higher intensity

\footnotetext{
t5 Work supported by US Department of energy, Division of High Energy Physics, contracts No. DE-ACO2-98CH10886 and DE-AC03-76SF00098.

*Corresponding author.

E-mail address: palmer@bni.gov (S. Ozaki).
}

(for physics reach). Fig. 1a has been adapted from a figure in a physics study [2]. Both studies were carried out under the auspices of the Muon Collider Collaboration [3] which has over 140 members from many institutions in the US and abroad. The components of the system are shown schematically in Fig. $1 \mathrm{~b}$.

\section{Components}

\subsection{Proton driver}

The proton driver is an upgrade (Fig. 2) of the Brookhaven Alternating Gradient Synchrotron (AGS) and uses many of the existing components and facilities. The current booster is replaced by a $1.3 \mathrm{GeV}$ Superconducting proton linac. The repetition rate is increased from 0.5 to $2.5 \mathrm{~Hz}$. The 

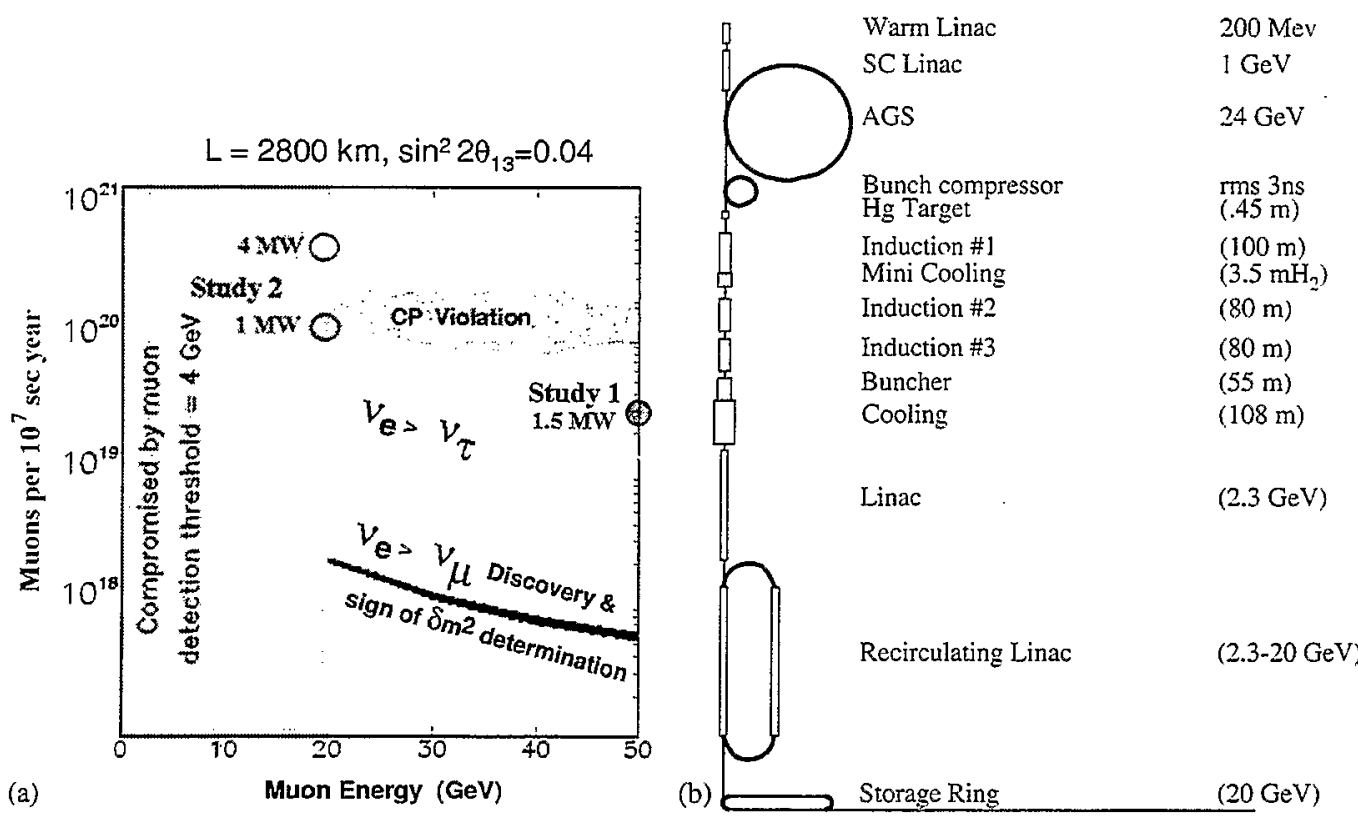

Fig. 1. (a) Muon decays in a straight section per $10^{7} \mathrm{~s}$ vs. muon energy, with fluxes required for different physics searches assuming a $50 \mathrm{kT}$ detector; together with the simulated performance of the two studies. (b) schematic of system.

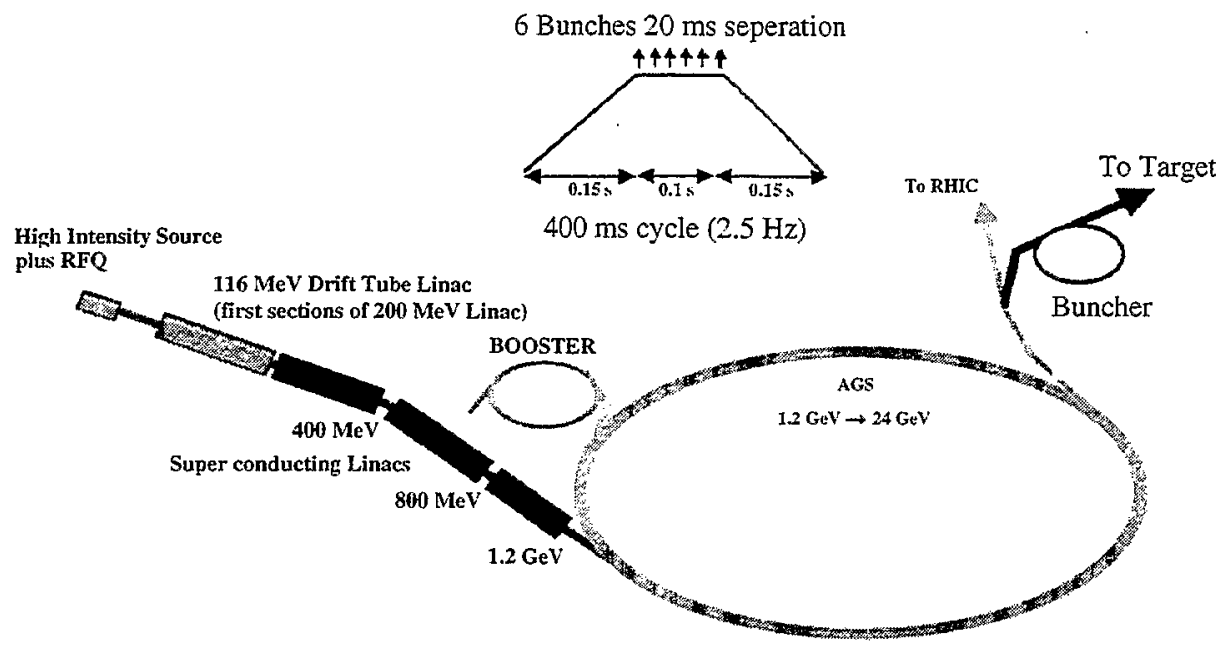

Fig. 2. Proton driver schematic.

total proton charge $\left(10^{14} \mathrm{ppp}\right)$ is only $30 \%$ higher than now. The 6 bunches would be extracted separately, spaced by $20 \mathrm{~ms}$, so that the target, induction linacs and RF systems that follow, need only be designed to deal with single bunches at an average repetition rate of $15 \mathrm{~Hz}$. The average power would be $1 \mathrm{MW}$. A possible upgrade to $2 \times$
$10^{14} \mathrm{ppp}$ and $5 \mathrm{~Hz}$ could give an average beam power of $4 \mathrm{MW}$.

\subsection{Target and capture}

A high $Z$ (mercury) target is chosen to give a high yield of pions per incident proton power 

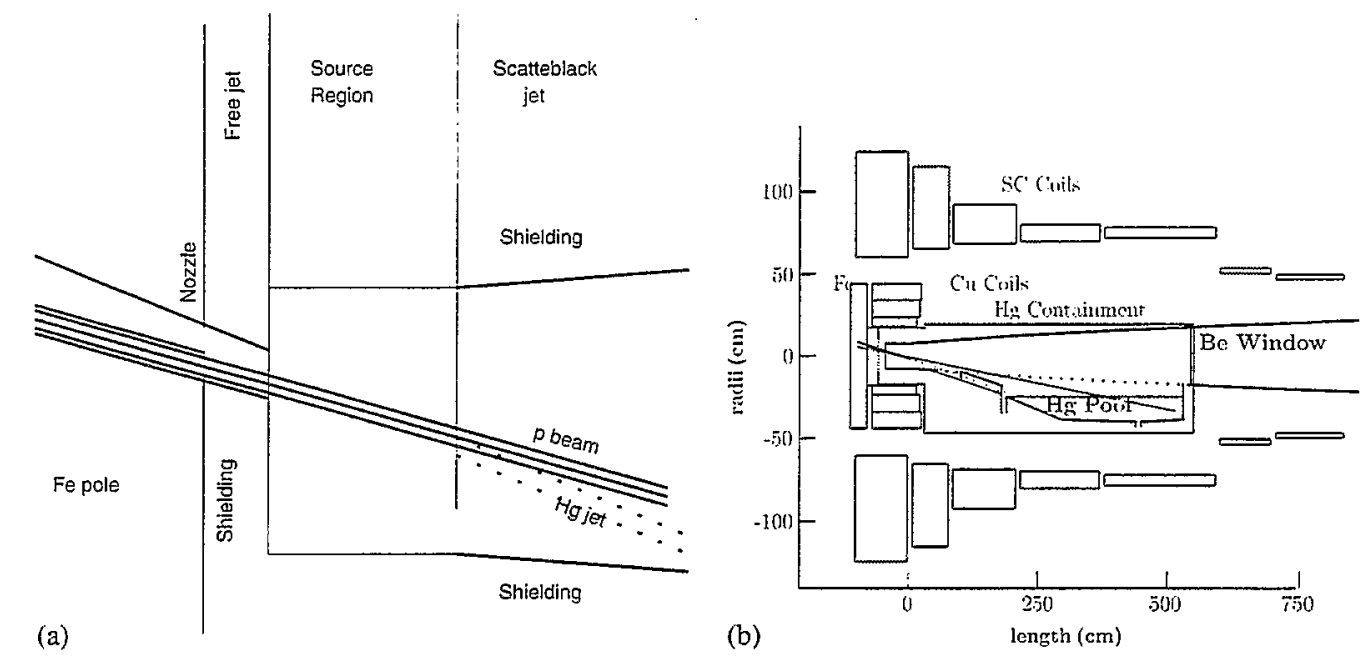

Fig. 3. (a) Mercury jet target geometry; (b) Mercury enclosure, mercury pool, beam dump, and solenoid capture magnets.

$(\approx 1.9 \times$ that for carbon). Solid metal targets could be used, but would need to be mechanically moved to avoid excessive temperatures and would have limited lifetime. The jet is continuous, is $1 \mathrm{~cm}$ diameter, and enters the target enclosure at $100 \mathrm{mrad}$ from the axis. The proton beam intersects the jet at an angle of $33 \mathrm{mrad}(67 \mathrm{mrad}$ from the axis). The geometry is shown in Fig. 3a. It is assumed that the thermal shock from the interacting proton bunch fully disperses the mercury. In this case the jet must have a velocity of $30 \mathrm{~m} / \mathrm{s}$ to be replaced before the next bunch. Perturbations to the jet by the capture magnetic field are controlled by placing the jet nozzle inside the field, so that the jet only sees $1 \mathrm{~T}$ field changes before it has passed beyond the production region.

Pions emerging from the target are captured and focused down the decay channel by a solenoidal field that is $20 \mathrm{~T}$ at the target center, and tapers down, over $18 \mathrm{~m}$, to a periodic $(50 \mathrm{~cm})$ superconducting solenoid channel (ave. field $\approx 1.25 \mathrm{~T}$ ) that continues through the phase rotation to the start of bunching. Fig. 3b shows a section of the $20 \mathrm{~T}$ hybrid magnet, the front end of the taper, the mercury containment, and mercury pool proton beam dump. The $20 \mathrm{~T}$ solenoid, with hollow copper insert and superconducting outsert, is not different in character to the higher field (up to $45 \mathrm{~T}$ ), but smaller bore, magnets at several existing labs. However, the copper insert in this design is made with hollow copper conductor and ceramic insulation to withstand radiation. MARS [4] simulations of radiation levels show that, with the shielding provided, both copper and superconducting magnets should have a lifetime greater than 20 years at $1 \mathrm{MW}$.

\subsection{Phase rotation}

Pions, and the muons into which they decay, are generated in the target over a very wide range of energies, but in a short time pulse (rms $3 \mathrm{~ns}$ ). This large energy phase is "rotated" into a longer time phase using drifts and induction linacs. The muons first drift to spread out their time, the induction linacs then decelerate the early ones and accelerate those later. 3 induction linacs (with lengths 100 , 80 , and $80 \mathrm{~m}$ ) are used in a system that reduces distortion [5] in the phase rotated bunch, and allows all induction units to operate with unipolar pulses. The $1.25 \mathrm{~T}$ beam transport solenoids are placed inside the induction cores to avoid saturating the ferrite. The induction units are similar to those being built for DARHT [6]. Fig. 4a shows the pulse shapes. Between the first and second induction linacs, two hydrogen absorbers (each $1.7 \mathrm{~m}$ long and $30 \mathrm{~cm}$ radius), with a field reversal between them, are introduced to reduce the transverse emittance (mini-cooling). Fig. 4b shows 

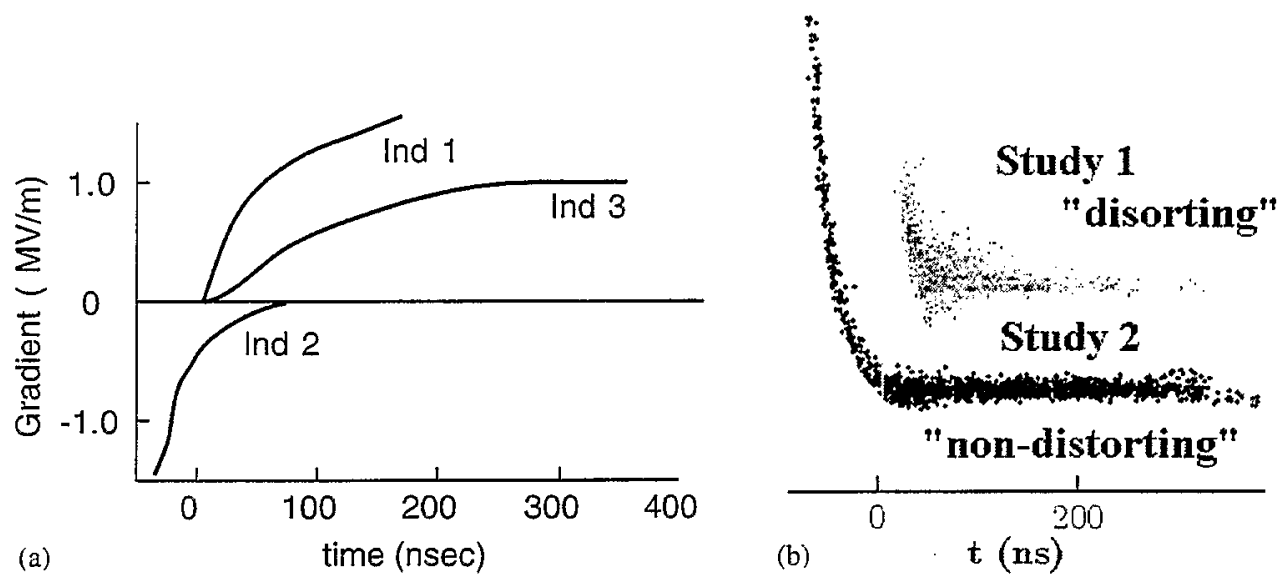

Fig. 4. (a) Pulse shapes of the 3 induction linacs; (b) Phase space after rotation for study 1 (above) and this study (below).

distribution of pions after the phase rotation for both study 1 (above) and this study (below). The study 1 (single induction unit) distribution shows the triangular "distorted" shape generated by relativistic non-linear effects. The distribution for this study (using more than one unit) shows relatively little distortion.

\subsection{Buncher}

The long bunch (400 $\mathrm{ns}$ ) after the phase rotation is bunched at $201 \mathrm{MHz}$ prior to cooling and acceleration at that frequency. The bunching is done in a lattice identical to that at the start of cooling (see below), and is preceeded by a matching section from the $1.25 \mathrm{~T}$ solenoids into this lattice. The bunching has three stages, each consisting of RF (with increasing acceleration) followed by a drifts with decreasing length. In the first two RF sections, second harmonic RF is used together with the $201 \mathrm{MHz}$ to improve the capture efficiency.

\subsection{Cooling}

Transverse emittance cooling is achieved by lowering the beam energy in hydrogen absorbers, interspersed with RF acceleration to keep the average energy constant. Transverse and longitudinal momenta are lowered in the absorbers, but only the longitudinal momentum is increased by the RF. Emittance increase from Coulomb scattering is minimized by maintaining the focus strength so that the angular spread of the beam is constant and large. To achieve this, the focus strength $\beta$ must be small and proportional to the emittance; i.e. falling as the emittance is cooled. A simple strong solenoid, with increasing field could achieve this, but such a field must anyway be reversed periodically to avoid a growth of angular momentum. Instead, a tapered alternating solenoid (SFOFO) [7] lattice is employed. The field shape is chosen to maximize the momentum acceptance $( \pm 22 \%)$ and provide $\beta$ 's (varying from 0.5 to $0.2 \mathrm{~m}$ ). Fig. $5 \mathrm{a}$ shows the initial axial field vs. length, and Fig. $5 \mathrm{~b}$ shows the $\beta$ functions vs. momentum at the start and end. Fig. $5 \mathrm{c}$ shows the simulated emittance as it falls as a function of length.

\subsection{Acceleration}

A $20 \mathrm{~m}$ SFOFO matching section, using conventional $R F$, raises the beta functions to $1 \mathrm{~m}$ prior to a $2.87 \mathrm{GeV}$ solenoid focused superconducting RF linac. This linac is followed by a single, recirculating linear accelerator (RLA) that raises the energy from 2.98 to $20 \mathrm{GeV}$, in 4 passes. The RLA has separate arcs for each pass, but the beam passes through the same linac on each pass. The arcs have an average radius ranging from 81 to $100 \mathrm{~m}$. The final arc has an average bending field of $0.59 \mathrm{~T}$. 

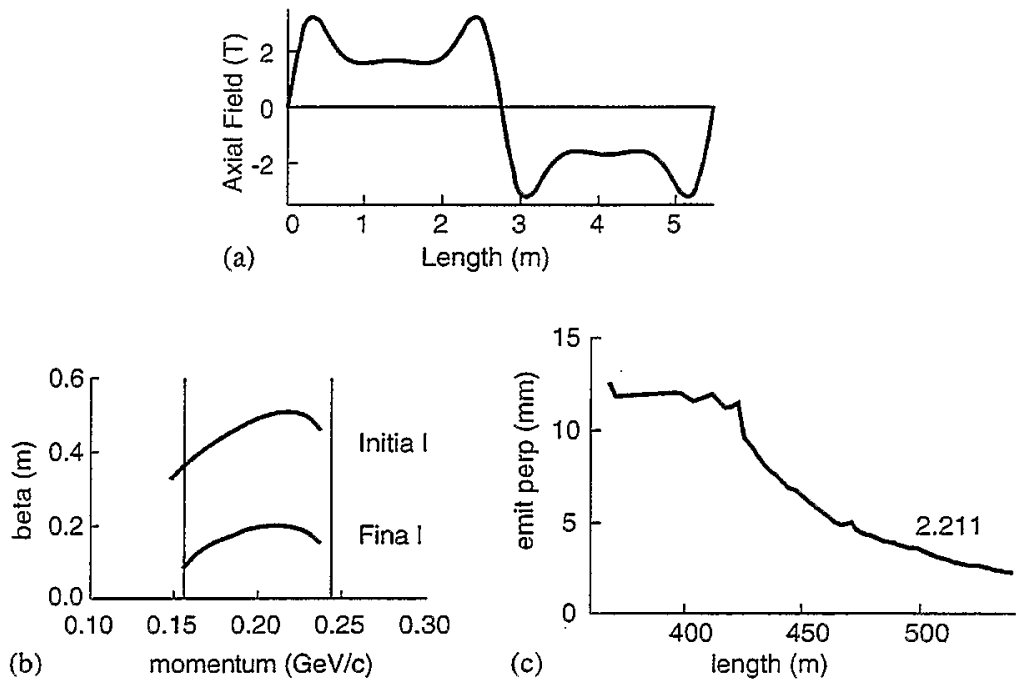

Fig. 5. SFOFO lattice. (a) Axial field vs. length; (b) $\beta$ functions vs. momentum at the start and end of the channel; (c) Transverse emittance vs. length in cooling.

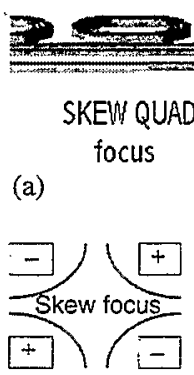

(b)
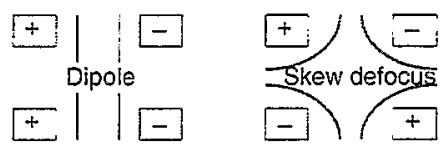

SKEWI QUAD defocus

Fig. 6. (a) Storage ring coils; (b) conceptual cross-sections of focus, bending, and defocus magnets; (c) Section through ring tunnel and cover.

\subsection{Storage ring}

After acceleration, the muons are injected into the upward straight of a racetrack shaped storage ring. High field superconducting arc magnets are used at each end to minimize the arc length and maximize the fraction ( $35 \%$ ) of muons that decay in the downward straight and generate neutrinos headed towards the detector at the WIPP facility in Carlsbad, $2903 \mathrm{~km}$ away. The muons, once injected, are never extracted, but are allowed to decay into electrons and neutrinos. The total heating from these electrons is $42 \mathrm{~kW}$ $(126 \mathrm{~W} / \mathrm{m})$. This load is too high to be dissipated in the superconducting coils. A magnet design has been chosen [8] that allows the majority of these electrons to pass out between separate upper and lower cryostats, and be dissipated in a dump at room temperature. In order to keep the midplane free of superconducting coils in the arcs, the lattice uses skew quadrupoles for focusing. In order to maximize the average bending field, $\mathrm{Nb}_{3} \mathrm{Sn}$ pancake coils are employed, and in order to maximize the packing factor, one coil of the bending magnet is extended and used as one half of the previous or following skew quadrupole (Fig. 6a). Fig. 6b shows a cross section of the ring which is kept above the water table and is covered by a $100 \mathrm{ft}$ high burm. The $300 \mathrm{ft}$ high BNL stack is also shown for scale. 


\section{Performance}

Complete simulations up to the start of acceleration have been performed by MARS [4] (for pion production) followed by ICOOL [9] (for transport, phase rotation and cooling). These have been confirmed by GEANT. They show 0.17 muons per initial proton on the target. i.e. 0.0071 $\mu$ 's/proton/proton energy in $\mathrm{GeV}$. This can be compared with a value of $0.0011 \mu$ 's/proton/proton energy in $\mathrm{GeV}$ in study 1 [1]. The gain $(\approx 6 \times)$ comes from: (a) The use of a mercury, instead of carbon, target $(1.9 \times)$; (b) From the use of three, instead of a single, phase rotation induction linacs (2x); (c) from the tapered cooling design (1.4x), and (d) from a larger accelerator acceptance $(1.2 \times)$. The muons delivered to the ring with a $1 \mathrm{MW}$ $(4 \mathrm{MW})$ proton driver, per $10^{7} \mathrm{~s}$, would be:

$$
\begin{aligned}
& 10^{14}(\mathrm{ppp}) \times 2.5(\mathrm{~Hz}) \times 10^{7}(\mathrm{~s}) \times 0.17(\mu / \mathrm{p}) \times 0.81(\mathrm{acc}) \\
& =3.4 \times 10^{20}\left(14 \times 10^{20}\right),
\end{aligned}
$$

and the number decaying in straight section would be:

$$
1.2 \times 10^{20}\left(4.8 \times 10^{20}\right) \text {. }
$$

\section{Conclusions}

This Study 2 shows significant improvements $(\approx 6 x)$ over Study 1 , and there remain possibilities of further gains. Cooling of the longitudinal emittance [10] and the capture of both signs [11] appear possible and, together might improve over- all performance by between 2 and 4 . More study is needed. In addition, development and technical work is required on many of the components.

\section{References}

[1] N. Holtkamp, D. Finley (Eds.), A feasibility study of a neutrino factory based on a muon storage ring, August 2000, http://www.fnal.gov/projects/muon_collider/nu-factory/fermi_study_after_aprillst/.

[2] C. Albright, et al., Physics at a Neutrino Factory, FERMILAB-FN-692, May 2000.

[3] J. Gallardo, http://www.cap.bnl.gov/mumu/.

[4] N.V. Mokhov, The MARS Code System User's Guide, Fermilab-FN-628 (1995), http://www-ap.fnal.gov/MARS/; N.V. Mokhov. Particle Yield and Radiation Fields in Feasibility Study-2 Target Capture System. MUC Note 0194. March 2001, http://www-mucool.fnal.gov/menotes/ muc0194.ps.

[5] R.B. Palmer, Non-Distorting Phase Rotation, MUC Note 0114, April 2000, http://www-mucool.fnal.gov/notes/.

[6] M.J. Burns, et al.. DARHT Accelerators Update and Plans for Initial Operation, Proceedings of the 1999 Accelerators Conference, 1999, p. 617.

[7] Eun-San Kim, et al., LBNL Report on Simulation and Theoretical Studies of Muon Ionization Cooling, MUC Note 0036, July 1999;

Eun-San Kim, M. Yoon, Super FOFO cooling channel for a Neutrino Factory, MUC Note 0191, February 2001, http://www-mucool.fnal.gov/notes/.

[8] A. Skrinsky, AIP Conf. Proc. 441 (1997) 249.

[9] R. Fernow, http://pubweb.bnl.gov/people/fernow/icool/.

[10] G. Hanson, http://needmore.physics.indiana.edu/"gail// emittance_exchange.html.

[11] D. Neuffer, High Frequency Buncher and $\phi-\delta E$ Rotation for the $\mu^{+}-\mu^{-}$Source, MUCOOL Note 0181, October 2000, http://www-mucool.fnal.gov/notes/. 